\title{
CAPÍTULO 37: TECNOLOGIAS EMERGENTES: UMA NOVA ABORDAGEM PARA REDUZIR SÓDIO E FOSFATO EM PRODUTOS CÁRNEOS
}

\section{EMERGING TECHNOLOGIES: A NEW APPROACH TO REDUCE SODIUM AND PHOSPHATE IN MEAT PRODUCTS}

\author{
Yasmim Sena Vaz Leães ${ }^{1}$; Mariana Basso Pinton²; Letícia Pereira Correa ${ }^{3}$; Alexandre José Cichoski ${ }^{4}$; \\ Paulo Cezar Bastianello Campagnol ${ }^{5}$
}

\section{Resumo}

Os aditivos são utilizados em produtos cárneos com diversas finalidades e, dentre os principais, estão o sal $(\mathrm{NaCl})$, que apresenta ação antimicrobiana, realça o sabor dos produtos, extrai e solubiliza proteínas; e o fosfato, que atua sinergicamente com o $\mathrm{NaCl}$, para reter a água do produto. Entretanto, o consumo em excesso de ambas substâncias está atrelado a diversos problemas de saúde, o que gera um problema de saúde pública e grande demanda por produtos com maior saudabilidade. A redução pode ocorrer de várias formas, mas pincipalmente por substituição parcial por outros sais, ou através de tecnologias verdes, tendo como principais a alta pressão hidrostática (APH), campo elétrico pulsado (CEP), ultrassom (US) e água eletrolisada básica (AEB). O trabalho objetivou destacar os principais e mais recentes estudos voltados à aplicação de APH, CEP, US e AEB como estratégia na redução de $\mathrm{NaCl}$ e fosfato em diferentes produtos cárneos, bem como explicar os princípios de cada uma das tecnologias e os possíveis fenômenos que possibilitaram a redução desses aditivos.

Palavras-Chave: alta pressão hidrostática, campo elétrico pulsado, ultrassom, água eletrolisada básica, tecnologias emergentes.

\begin{abstract}
Additives are used in meat products for various purposes. The main ones are salt $(\mathrm{NaCl})$, which has an antimicrobial action, improve the flavor of the products, extracts and solubilizes proteins; and phosphate, which acts synergistically with $\mathrm{NaCl}$, to retain the product water. However, the excessive consumption of both substances is linked to several health problems, which generates a public health problem and great demand for products with greater health. The reduction can occur in several ways, but mainly by partial replacement with other salts, or through green technologies, with high hydrostatic pressure (HHP), pulsed electric field (PEF), ultrasound (US) and basic electrolyzed water as main (BEW). The aim of this work was to highlight the main and most recent studies focused on the application of APH, CEP, US and AEB as a strategy to reduce $\mathrm{NaCl}$ and phosphate in different meat products, as well as explaining the principles of each of the technologies and the possible phenomena that enabled the reduction of these additives.
\end{abstract}

Keywords: high hydrostatic pressure, pulsed electric field, ultrasound, basic electrolyzed water, emerging technologies.

\section{Introdução}

\footnotetext{
${ }^{1}$ Doutoranda em Ciência e Tecnologia dos Alimentos, Universidade Federal de Santa Maria, yasmimsvl@ hotmail.com

${ }^{2}$ Doutoranda em Ciência e Tecnologia dos Alimentos, Universidade Federal de Santa Maria, mbpinton@gmail.com

${ }^{3}$ Graduanda em Tecnologia dos Alimentos, Universidade Federal de Santa Maria, pereiracorreal@gmail.com

${ }^{4}$ Doutor em Tecnologia de Alimentos (UFPR), Professor da Universidade Federal de Santa Maria, cijoale@ gmail.com

5 Doutor em Tecnologia de Alimentos (UNICAMP), Professor da Universidade Federal de Santa Maria, paulocampagnol@gmail.com
} 
LEÃES, Y.S.; PINTON, M. B.; CORREA, L.P.; CICHOSKI, A.J.; CAMPAGNOL, P. C. B.

Aditivos são utilizados em produtos cárneos com as mais diversas finalidades, seja para a segurança do alimento, a conservação (efeito antioxidante e antimicrobiano), adquirir características específicas de cor, textura, sabor, aroma, ou para conferir características tecnológicas que afetam diretamente o rendimento do produto final, ao evitar que ocorram perdas de frações aquosas e/ou lipídicas. Porém, sabe-se que o consumo excessivo desses aditivos está relacionado ao aumento nos problemas de saúde, fazendo-se necessário encontrar alternativas que proporcionem sua redução e promovam o consumo de produtos com maior saudabilidade, ao mesmo tempo em que nenhuma das características citadas sejam afetadas (THANGAVELU et al., 2019). Nesse sentido, o aditivo mais estudado atualmente quanto alternativas para sua redução é o sal $(\mathrm{NaCl})$. $\mathrm{O}$ fosfato, apesar de recente, também vem ganhando espaço nesses estudos (PINTON et al., 2021).

$\mathrm{O} \mathrm{NaCl}$ é um ingrediente cujas funcionalidades influenciam diretamente na qualidade microbiológica, sensorial (como realçador de sabor) e, sobretudo, tecnológica de produtos cárneos. Os íons $\mathrm{Cl}^{-}$presentes no $\mathrm{NaCl}$ doam as cargas negativas às proteínas, fazendo com que elas se afastem e assim retenham mais água, o que confete maior rendimento ao produto final. Entretanto, o sódio contido em sua molécula, quando consumido em excesso, está atrelado à diversos problemas de saúde, tais como aumento no risco de doenças cardiovasculares, infarto, acidente vascular cerebral, obesidade, etc (INGUGLIA et al., 2017).

$\mathrm{O}$ fosfato atua sinergicamente com o $\mathrm{NaCl}$ na extração e solubilização das proteínas, bem como no aumento da capacidade de retenção de água (CRA) ao promover aumento no $\mathrm{pH}$ do produto e consequente distanciamento do ponto isoelétrico (PI). Além disso, ele também possui atividade antioxidante sobre os produtos cárneos. Porém, ele é de especial preocupação ao grupo de pessoas com problemas renais, uma vez que afeta a capacidade de expelir ácidos ocasionando um acúmulo nos níveis de fosfato, o que ocasiona no aumento de até $40 \%$ na taxa de mortalidade dessas pessoas. Além disso, em pessoas que não possuem problemas renais, ele prejudica a absorção de cálcio, ocasionando a maior propensão a doenças ósseas (PINTON et al., 2019).

Desta forma, entende-se que há uma demanda cada vez maior pela saudabilidade desses produtos, o que implica na redução desses aditivos. Acerca disso, os estudos envolvendo a redução de $\mathrm{NaCl}$ e fosfato são contemplados principalmente por dois segmentos atualmente: redução por substituição parcial por outros sais $\left(\mathrm{KCl}, \mathrm{CaCl}_{2}\right.$, ou $\mathrm{MgCl}_{2}$ ); e/ou pela aplicação de novas tecnologias, sendo estas capazes de promoverem modificações na estrutura da matriz alimentar de forma a compensar a redução desses compostos no produto. Dentre as novas tecnologias estudadas objetivando redução de 
LEÃES, Y.S.; PINTON, M. B.; CORREA, L.P.; CICHOSKI, A.J.; CAMPAGNOL, P. C. B.

$\mathrm{NaCl}$ e fosfato, as mais utilizadas são a alta pressão hidrostática (APH), o campo elétrico pulsado (CEP), o ultrassom (US) e, recentemente, a água eletrolisada básica (AEB) em conjunto com o ultrassom (BHAT et al., 2020; LEÃES et al., 2020; PINTON et al., 2020; PINTON et al., 2019; WANG et al., 2018).

Elas são consideradas "tecnologias verdes" ou "tecnologias emergentes" por apresentarem características ecologicamente corretas, uma vez que otimizam o uso de recursos naturais, economizam a energia utilizada e reduzem custos na indústria de alimentos, e vem ganhando cada vez mais espaço em relação às técnicas convencionais de processamento e conservação (ALARCÓN-ROJO, 2019; FLORES et al., 2018). Essas tecnologias vêm apresentando resultados positivos na redução de $\mathrm{NaCl}$ e fosfato, sem necessariamente utilizar da substituição parcial por outros sais. Isso porque elas promoveram ação sobre as proteínas da carne, principais responsáveis pela retenção de água e consequente manutenção da textura em diversos produtos cárneos (CICHOSKI et al., 2019).

Desta forma, o presente trabalho objetivou destacar os principais e mais recentes estudos voltados à aplicação de APH, CEP, US e AEB como estratégia na redução de $\mathrm{NaCl}$ e fosfato em diferentes produtos cárneos, bem como explicar os princípios de cada uma das tecnologias e os possíveis fenômenos que possibilitaram a redução desses aditivos.

\section{Alta Pressão Hidrostática (APH)}

O tratamento com alta pressão hidrostática é considerado um processo não térmico, alternativo aos tratamentos térmicos, permitindo a inativação de microrganismos patogênicos e deteriorantes nos alimentos (JÚNIOR et al., 2019). Aumenta o prazo de validade dos produtos, pois atua como um processo de pasteurização a frio, afetando minimamente os atributos sensoriais e nutricionais dos alimentos (ANDREOU et al., 2017).

A aplicação de alta pressão é baseada em dois princípios que determinam o comportamento dos alimentos sob pressão. O princípio Le Châtelier, princípio importante subjacente aos efeitos no equilíbrio da reação. Qualquer fenômeno acompanhado por uma diminuição no volume será aprimorado por um aumento da pressão e vice-versa. Transições de fase associadas com uma diminuição de volume são afetadas, enquanto as acompanhadas com aumento de volume são inibidas (GUILLOU et al., 2017). A aplicação de pressão leva a alterações físicas e químicas. Aumento na pressão contribui para uma diminuição na distância intermolecular média entre as moléculas. A compressão física durante o tratamento de alta pressão resulta em um aumento de temperatura através 
LEÃES, Y.S.; PINTON, M. B.; CORREA, L.P.; CICHOSKI, A.J.; CAMPAGNOL, P. C. B.

do aquecimento adiabático $\pm 3{ }^{\circ} \mathrm{C}$ por $100 \mathrm{MPa}$, dependendo da composição dos alimentos. Já as alterações químicas são mínimas. Somente ligações não covalentes, como hidrogênio e ligações iônicas, interações hidrofóbicas nos alimentos são interrompidas pela pressão (POTTIER et al., 2017; GUILLOU et al., 2017). O segundo princípio associado aos efeitos da alta pressão é o princípio isostático, que afirma que a pressão é instantaneamente e uniformemente transmitida por todo o produto sem gradiente de pressão. Deste modo, a pressão é caracterizada como pressão isostática.

O processo de alta pressão opera descontinuamente em um produto embalado (geralmente sob vácuo) para evitar os riscos de contaminação após o tratamento. A embalagem deve ser flexível para suportar até $600 \mathrm{MPa}$. O produto é introduzido no recipiente de tratamento cilíndrico, normalmente horizontal, com um volume interno variando de 50 a $525 \mathrm{~L}$ para dispositivos industriais atualmente disponíveis comercialmente. $\mathrm{O}$ vaso é preenchido com um fluido transmissor de pressão (geralmente água) e depois pressurizado usando uma bomba de alta pressão variando de 100 a 600 $\mathrm{MPa}$, e as vezes até $900 \mathrm{MPa}$. A pressão aplicada diretamente, por um sistema de pistão, ou indiretamente, por um sistema de bomba hidráulica, é transmitida isostaticamente dentro do vaso de pressão, permitindo que o alimento seja instantaneamente e uniformemente tratado independente do volume e da geometria. A pressão é mantida por um tempo de espera, geralmente 1 - 5 minutos, e, em seguida, a abertura de uma válvula causa despressurização quase instantânea do vaso. Todo o processo é realizado a uma temperatura de 4 a $20{ }^{\circ} \mathrm{C}$ (POTTIER et al., 2017, SOLADOYE; PIETRASIK, 2018). O processo de alta pressão apresenta diversas vantagens em relação a outras tecnologias não térmicas, como baixo consumo de energia, baixo risco de contaminação, e, além disso, é uma tecnologia de processamento ecológica (HUANG et al., 2017).

\section{Efeito da APH nos produtos cárneos para redução de aditivos}

As indústrias cárneas têm como objetivo prolongar a vida útil de carnes e produtos cárneos sem uso de conservantes químicos e sem afetar as qualidades sensoriais. A tecnologia de alta pressão pode oferecer algumas oportunidades para atingir esse objetivo. Muitos estudos demonstraram que o tratamento de alta pressão pode ser usado para produzir produtos cárneos com baixo teor de aditivos como, por exemplo, sódio e fosfato (O’FLYNN et al., 2014; O’NEILL et al., 2015; TAMM et al., 2016; PIETRASIK et al., 2016).

Recentemente, Pietrasik et al. (2017) utilizaram APH (600 MPa, 3 minutos, $8{ }^{\circ} \mathrm{C}$ ) para avaliar a qualidade de salsichas com redução ou substituição de $50 \%$ de $\mathrm{NaCl}$ por $\mathrm{KCl}$. Os autores reportaram que o uso de APH estendeu a vida útil das salsichas por até 
LEÃES, Y.S.; PINTON, M. B.; CORREA, L.P.; CICHOSKI, A.J.; CAMPAGNOL, P. C. B.

12 semanas e melhorou a textura devido a melhorar a retenção de umidade. Já O’Neil et al. (2018) utilizaram uma combinação de substitutos de sal (Artisalt TM - teor de redução de 0,50 e 100\%), APH $(0,300,600 \mathrm{MPa})$ e antimicrobianos (Inbac TM teor de 0,2, 0,3 e $0,4 \%$ ) no desenvolvimento de presunto cozido. A substituição de 50\% do substituto de sal não causou alterações nos atributos testados. O estudo mostrou que a melhor combinação das variáveis testadas para o desenvolvimento de presunto cozido com baixo teor de sal é de $54 \%$ de substituição de sal, uso de $535 \mathrm{MPa}(\mathrm{APH})$ e $0,3 \%$ de concentração de Inbac TM.

Zheng et al. (2019) utilizaram alta pressão (200 MPa) e reduziram a quantidade de sal em linguiça de frango. Os resultados mostraram efeitos benéficos na qualidade tecnológica de linguiças como textura relativamente mais resistente e elástica e diminuição na perda de cozimento. No estudo de Wang et al. (2018) foi combinado APH (200 MPa) com baixos níveis de $\mathrm{CaCl}_{2}(<40 \mathrm{mM})$ aumentando a solubilidade das proteínas miofibrilares, o que garantiu propriedades funcionais no peito de frango.

De acordo com os estudos citados, é possível produzir produtos cárneos com concentrações reduzidas de sal utilizando alta pressão, sem que ocorra a perda das propriedades tecnológicas, sensoriais e microbiológicas e ainda, aumentando a vida útil. O uso da tecnologia de alta pressão hidrostática pode aumentar o desdobramento das proteínas e a funcionalidade de ligação de água, havendo potencial para aplicação em formulação com quantidade reduzida de sal. A tecnologia de alta pressão é uma tecnologia viável que pode compensar parcialmente a redução de sal, atendendo as qualidades tecnológicas dos produtos e também a segurança microbiológica.

\section{Campo Elétrico Pulsado (CEP)}

Em meados do século XX, começou a ser aplicado o tratamento por campo elétrico pulsado (CEP) para processamento de alimentos e produtos agrícolas (SITZMANN et al., 2016).

O CEP garante a segurança microbiana do produto sem afetar significativamente o valor organoléptico e nutricional do alimento sendo processado, devido ao acúmulo mínimo de calor durante o tratamento (BEKHIT et al., 2017), o que garante a produção de produtos de qualidade.

Essa tecnologia envolve o uso de pulsos de campo elétrico de curta duração (variando de nanossegundos a milissegundos) com força de campo elétrico de 0,1 a 80 $\mathrm{kV} / \mathrm{cm}$ aplicada a um alimento entre dois eletrodos (BHAT et al., 2018). Tem potencial de substituir o processamento térmico tradicional, como por exemplo, a pasteurização, o 
LEÃES, Y.S.; PINTON, M. B.; CORREA, L.P.; CICHOSKI, A.J.; CAMPAGNOL, P. C. B.

que pode melhorar a eficiência do processo e a qualidade do produto final (BEKHIT et al., 2017).

O CEP provoca alterações estruturais locais e uma rápida quebra da membrana celular que pode ser permanente ou temporária, dependendo da intensidade e das condições de tratamento. Deste modo, o tratamento do CEP consiste na aplicação de um campo elétrico externo que leva à quebra da membrana celular, induzindo mudanças estruturais. Esse fenômeno é conhecido como eletroporação ou eletro-permeabilização (TOEPFL et al., 2014). A eletroporação depende de muitos fatores, como parâmetros do CEP (amplitude do pulso, número e duração dos pulsos elétricos), propriedades celulares (tamanho, forma, orientação) e parâmetros da membrana (temperatura, força iônica) (SAULIS, 2010). Os parâmetros do processo do CEP podem ser ajustados dependendo da aplicação desejada. A diversidade de matérias-primas e a disponibilidade de equipamentos de CEP podem afetar as condições de processo (GÓMEZ et al., 2019).

A eletroporação envolve a formação de poros e aumenta a permeabilidade das membranas biológicas (OSTERMEIER et al., 2018). Essa permeabilidade aumentada da membrana facilita a troca de componentes intracelulares com o ambiente da célula e pode induzir certos efeitos úteis que não foram totalmente explorados (BHAT et al., 2018), como por exemplo, a melhor difusão de sal, extração de compostos ou alterações nas propriedades físicas.

\section{Efeito do CEP na difusão de aditivos em produtos cárneos}

Existem diversas pesquisas de aplicação de CEP em bebidas alcóolicas (YANG et al., 2016; RICCI et al., 2018), produtos lácteos (BUCKOW et al., 2014; SHARMA et al., 2014) e ovos de aves (ESPINA et al., 2014; YOGESH, 2016) e em controle de microrganismos (BARBA et al., 2017; GABRIC et al., 2017).

O tratamento com CEP também pode ser uma boa estratégia para alterar as propriedades em produtos cárneos, (BEKHIT et al., 2016). De acordo com Gómez et al. (2019) a absorção das substâncias comuns usadas nos períodos de cura dos produtos cárneos, como sal, nitritos e especiarias, poderia ser melhorada após o tratamento com $\mathrm{CEP}$, devido à ruptura parcial dos tecidos celulares e seu efeito nos processos de transferência de massa. No entanto, ainda são poucos os estudos relacionados com CEP e aditivos alimentares.

Contudo, Bhat et al (2020) comprovaram o uso de CEP na redução de sódio em músculo Semimembranosos, onde o tratamento permitiu uma redução direta de $\mathrm{NaCl}$ com uma melhor difusão do sal na matriz da carne, sem danos na qualidade sensorial e estabilidade oxidativa e microbiana dos produtos. Autores como McDonnell et al. (2014) 
LEÃES, Y.S.; PINTON, M. B.; CORREA, L.P.; CICHOSKI, A.J.; CAMPAGNOL, P. C. B.

demonstraram que há potencial de aceleração de salga em carne suína (M. Longissimus thoracis e Lumborum LTL). Comprovaram uma maior perda de cozimento e efeitos na textura, como aumento da dureza e mastigação, talvez pelo aumento da solubilização das fibras devido a maior concentração de $\mathrm{NaCl}$, associado ao aumento da perda de água durante o cozimento. Os parâmetros utilizados foram: força de campo $\left(1,2 \mathrm{Kv} / \mathrm{cm}^{3}\right)$, frequência $(100 \mathrm{~Hz})$, número de pulso (300 pulsos) e tempo (3 ms) e, ainda, atuaram para o aumento da difusão salina $\left(175 \mathrm{~kg} / \mathrm{m}^{3} \mathrm{de} \mathrm{NaCl}\right)$.

\section{Ultrassom (US)}

O ultrassom é uma onda sonora em uma frequência que excede o limiar da audição humana $(20 \mathrm{kHz})$. É uma forma de energia vibracional produzida por um transdutor de ultrassom que converte energia elétrica em energia acústica (CHEN et al., 2020), portanto, é uma forma de energia mecânica não ionizante, não invasiva e não poluente. É considerado um método emergente com um grande potencial para controlar, melhorar e acelerar processos sem prejudicar a qualidade dos alimentos (ALARCON-ROJO et al., 2019), alterando as propriedades físicas, químicas e funcionais.

Em um sistema de ultrassom, a energia elétrica é transformada em energia vibracional, que pode induzir cavitação. A energia da cavitação produz efeitos químicos, físicos ou biológicos. Vários estudos relataram os efeitos do ultrassom no processamento de carne, alterando as propriedades físicas, químicas e funcionais, podendo auxiliar na cura, maciez e, ainda, beneficiar na redução de aditivos químicos (INGUGLIA et al., 2017).

O ultrassom é uma onda sonora caracterizada por vários parâmetros como a frequência (número de ciclos repetidos por unidade de tempo), a mudança na onda sonora (deslocamento máximo de partículas ou nível máximo do sinal acústico) durante um único período de tempo (amplitude), a distância entre pontos correspondentes consecutivos da mesma fase (comprimento de onda), a velocidade de propagação da onda (velocidade do som) e a perda gradual de intensidade do som através de um meio (coeficiente de atenuação) (NOWAK et al., 2017). O US é dividido em três regiões de frequência, todas acima do limiar da audição humana. O ultrassom de potência é de 16 a $100 \mathrm{kHz}$. O ultrassom de alta frequência é de $100 \mathrm{kHz}$ a $1 \mathrm{MHz}$, enquanto que o ultrassom de diagnóstico é de 1 a $10 \mathrm{MHz}$ (INGUGLIA et al., 2017).

A base das aplicações do ultrassom na faixa de frequência de $20 \mathrm{kHz}$ a $1 \mathrm{MHz}$ é a cavitação acústica, que ocorre em regiões sob ondas de alta amplitude que se alternam rapidamente e consiste no crescimento e colapso de bolhas de gás em um meio líquido, resultando em modificações físicas dos tecidos musculares (GONZALES-GONZALEZ 
LEÃES, Y.S.; PINTON, M. B.; CORREA, L.P.; CICHOSKI, A.J.; CAMPAGNOL, P. C. B.

et al., 2020). Portanto, a cavitação é a formação de bolhas na fase líquida quando a pressão negativa é aplicada e a distância entre as moléculas é pelo menos o dobro da distância de van der Waals. Quando a bolha é exposta a mudanças na pressão acústica, dois possíveis cenários podem ocorrer: a bolha entra em um ciclo continuo de expansão-compressão (estado conhecido como cavitação estável) ou a bolha cresce para um tamanho crítico pelo qual ela entra em colapso instantaneamente (cavitação transitória) (NOWAK et al., 2017). Bolhas estáveis são consideradas bolhas fracamente e simetricamente oscilantes, enquanto que bolhas transitórias são consideradas bolhas de cavitação ativa. O colapso das bolhas é um processo quase adiabático e resulta na geração de altas temperaturas (> $\left.5000{ }^{\circ} \mathrm{C}\right)$ e altas pressões (300 - $\left.1000 \mathrm{~atm}\right)$ (NOWAK et al., 2017).

A cavitação produz forças físicas intensas que incluem forças de cisalhamento, ondas de choque e turbulências, alterando potencialmente as propriedades funcionais das proteínas por métodos físicos ou químicos. Durante a sonicação, as bolhas são formadas rapidamente e colapsam intensamente, o que pode ser um dos motivos das alterações químicas e físicas. $\mathrm{O}$ aumento da pressão e temperatura nas proximidades dessas cavidades é à base das mudanças no meio exposto ao ultrassom (AMIRI et al., 2018). Nos produtos cárneos, os microjatos, causados por bolhas implodentes, colidem com a superfície das miofibrilas, causando micro fissuras e alterando a estrutura dos produtos (BARRETO et al., 2018). Siró et al. (2009) explica que a cavitação causada pelo ultrassom abre canais entre as miofibrilas, o que facilita a penetração de salmouras, melhorando assim, a difusão dos aditivos.

\section{Efeito do US na redução de aditivos nos produtos cárneos}

Barreto et al. (2018) avaliaram os efeitos da redução de sal com o auxílio do US em presuntos cozidos. O ultrassom reduziu a liberação total de fluidos, os presuntos apresentaram uma maior capacidade de retenção de água, o que resultou na melhoria dos parâmetros de textura e aceitação sensorial.

Pela primeira vez, a cinética de difusão de salmoura ativada no interior da carne assistida por ultrassom foi estudada por Krasulya et al. (2019). De acordo com esses autores, as salmouras com diferentes concentrações de $\mathrm{NaCl}$ à base de água eletro ativada, tem velocidade de propagação diferente. Quanto maior a concentração de salmoura, mais intensivo é o processo de penetração de substâncias salgadas nas amostras, portanto, o uso de salmoura ativada por cavitação pode contribuir para uma melhor difusão dos ingredientes e aceleração dos processos físico-químicos e bioquímicos que ocorrem durante o tratamento da salmoura reduzindo o tempo de cura da carne. Outro ponto importante é o tempo de cura, em um quatro dias de cura, a salmoura tratada permeou 
LEÃES, Y.S.; PINTON, M. B.; CORREA, L.P.; CICHOSKI, A.J.; CAMPAGNOL, P. C. B.

toda a profundidade do músculo com $3 \%$ de $\mathrm{NaCl}$. Pode-se argumentar que o uso de salmoura ativada por cavitação pode reduzir o tempo de salmoura da carne.

Cichoski et al. (2019) avaliaram atributos tecnológicos em emulsões cárneas submetidas a diferentes modos de operação do ultrassom, como os modos operacionais normal, degas e sweep. De acordo com os resultados, o uso de $60 \%$ de amplitude, $25 \mathrm{kHz}$ de frequência e modo normal foram os parâmetros mais eficazes para melhoria da qualidade tecnológica das emulsões cárneas. Esses resultados deram embasamento para outros estudos utilizando ultrassom como estratégia para redução de aditivos químicos, como os citados abaixo.

Pinton et al. (2019) avaliaram o efeito do ultrassom na redução de fosfatos em emulsões cárneas. Provaram que com o uso de 18 minutos de tratamento de US $(60 \%$ amplitude, frequência de $25 \mathrm{kHz}$, potência nominal de $1000 \mathrm{~W}$, modo normal) aumentou o rendimento de cozimento com redução de $50 \%$ de fosfato, e ainda, melhorou a estabilidade de emulsão. Leães et al (2020) utilizaram ultrassom em diferentes tempos (10 e $20 \mathrm{~min}$ ) para reduzir $\mathrm{NaCl}$ em produtos cárneos emulsionados. Estes autores obtiveram resultados semelhantes em relação à estabilidade da emulsão e rendimento de cozimento, com reduções de até $20 \%$ de $\mathrm{NaCl}$ no tempo de 20 minutos de ultrassom sem afetar a qualidade tecnológica do produto quando comparado ao padrão. Em estudo mais recente realizado por Pinton et al. (2020), foi investigada a ação do US em conjunto com diferentes sais substitutos de $\mathrm{NaCl}\left(\mathrm{KCl}, \mathrm{CaCl}_{2}\right.$, or $\left.\mathrm{MgCl}_{2}\right)$ em emulsões cárneas com teor reduzido de sal e fosfato. Através desse estudo, foi possível reduzir cerca de $50 \%$ do $\mathrm{NaCl}$ utilizando a combinação de 27 min de US e $0,5 \%$ de $\mathrm{KCl}$, sem comprometer características físico-químicas, tecnológicas e oxidativas.

Estes resultados na redução de fosfato e sal nas emulsões estão atrelados a, basicamente, três fatores: 1) a modificação na estrutura das proteínas promovidas pelo US, permitindo a exposição das cadeias laterais polares e apolares dos aminoácidos (permitindo a ligação da água e da gordura); 2) a melhor difusão de fosfato e $\mathrm{NaCl}$ na massa; 3) e a redução e padronização do glóbulo de gordura, evitando assim que eles coalesçam e se separem da fase aquosa, aspecto importante na estabilidade de uma emulsão. Assim, quando cozidas, também apresentaram efeitos positivos na estabilidade do gel oriundo da agregação proteica promovida pelo cozimento, o que influenciou diretamente na textura do produto (CICHOSKI et al., 2019; INGUGLIA et al., 2017; CHEMAT, 2011).

\section{Água eletrolisada (AE)}


LEÃES, Y.S.; PINTON, M. B.; CORREA, L.P.; CICHOSKI, A.J.; CAMPAGNOL, P. C. B.

A obtenção da AE ocorre através do processo de eletrólise, que consiste na passagem de uma solução salina (geralmente $\mathrm{NaCl}$ ) por uma câmara dotada de uma célula de eletrolisação. Essa célula geralmente é separada por uma membrana, originando dois polos: o ânodo (-) e o cátodo (+), os quais originam dois produtos distintos: a água eletrolisada ácida (AEA) e a água eletrolisada básica (AEB), respectivamente (ATHAYDE et al., 2018; HUANG et al., 2008).

Durante a eletrólise, ocorre migração dos íons $\mathrm{Cl}^{-}$para o ânodo e dos íons $\mathrm{Na}^{+}$ para o cátodo. Os principais produtos da $\mathrm{AEA}$ são $\mathrm{Cl}_{2}$ dissolvido, ácido hipocloroso $(\mathrm{HOCl})$ e ácido clorídrico $(\mathrm{HCl})$ diluído, e esta possui um $\mathrm{pH}$ ácido $(<3.0)$ e potencial de oxirredução $(\mathrm{POR})>1000 \mathrm{mV}$. A AEB tem como principais produtos o hidróxido de sódio $(\mathrm{NaOH})$, uma pequena quantidade de cloro na forma de hipoclorito $\left(\mathrm{OCl}^{-}\right)$e também $\mathrm{H}_{2}$, tendo como principal característica o pH alcalino (> 10.0) e POR de -800 a $900 \mathrm{mV}$ (CUI et al., 2009).

Entretanto, um dos maiores problemas em relação à AEA está a sua instabilidade (uma vez que o cloro se apresenta na forma de gás) e sua corrosividade. Desta forma, a fim de contornar esses fatores, há a produção de um terceiro tipo de água: a água eletrolisada fracamente ácida (AEFA), gerada a partir da mistura de AEA e AEB, sem a separação do ânodo e cátodo por membrana. Essa água possui $\mathrm{pH}$ mais próximo da neutralidade, entre 6,0 e 6,5 e POR de 800 a $900 \mathrm{mV}$, apresentando cerca de $95 \%$ do cloro na forma de ácido hipocloroso $(\mathrm{HClO}), 5 \%$ na forma de íon hipoclorito $\left(\mathrm{OCl}^{-}\right)$e traços de $\mathrm{Cl}_{2}$ (AFARI; HUNG, 2018; ATHAYDE et al., 2018).

Os estudos realizados na área de alimentos utilizaram concentrações de salmoura que variaram de 0,01 a 5\%, para diferentes matrizes alimentares e finalidades. Diversos trabalhos evidenciaram a eficácia da $\mathrm{AE}$ em processos de sanitização, já tendo sido aplicada em hortaliças, carne de frango, carne suína e superfícies de manipulação de alimentos e tendo efeito sobre diversos microrganismos patogênicos e deteriorantes, como Listeria monocytogenes, Escherichia coli, Salmonella, Shewanela, coliformes, mesófilos e psicrotróficos em diferentes matrizes alimentares (WANG et al., 2019; WANG et al., 2018; AFARI; HUNG, 2018; SHENG et al., 2018; ATHAYDE et al., 2017; LI et al., 2016; SHIMAMURA et al., 2016; OVISSIPOUR et al., 2015; LEE et al., 2014; SUN et al., 2012). Essas variações na concentração são decorrentes de que as propriedades físico-químicas e efeitos da $\mathrm{AE}$ variam de acordo com a concentração $\mathrm{NaCl}$, tempo de eletrólise ou fluxo de água (ATHAYDE et al., 2018; RHAMAN; KHAN; OH, 2010).

Essa eficácia ocorre devido à presença do cloro, mais predominante na AEA e AEFA, as quais são estudadas em separado ou combinadas também com AEB a fim de 
LEÃES, Y.S.; PINTON, M. B.; CORREA, L.P.; CICHOSKI, A.J.; CAMPAGNOL, P. C. B.

avaliar a eficácia de ação antimicrobiana. Algumas vantagens em relação a outros agentes sanitizantes tradicionais, são a desinfecção eficaz, fácil operação, baixo custo e ambientalmente correta (JADEJA; HUNG, 2014).

Em relação a esta tecnologia, embora o equipamento de eletrólise tenha um custo elevado, a produção da $\mathrm{AE}$ é simples e há possibilidade de diversas adaptações na aplicação, podendo ser utilizada na forma de spray, por imersão ou gelo, constituindo-se de um processo simples, com eficiente capacidade antimicrobiana (ATHAYDE et al., 2018; HUANG et al., 2008; SUN et al., 2012). Assim, constata-se que a maior parte dos estudos são voltados ao uso dos diferentes tipos de AE em processos de sanitização na indústria de alimentos, objetivando redução ou eliminação de carga microbiana, sendo a AEA ou a AEFA mais explorada nesses estudos. Em relação à $\mathrm{AEB}$, são poucos os estudos que tratam de sua aplicação em alimentos, e em boa parte esta é aplicada em combinação com AEA e AEFA a fim de potencializar a ação antimicrobiana.

\section{Aplicação da AEB em alimentos e seu uso como estratégia na redução de NaCl em produtos cárneos}

A AEB possui características que não são necessariamente voltadas à ação antimicrobiana, porém ainda são pouco exploradas. Seu pH alcalino e o $\mathrm{NaOH}$ contido nela podem conferir modificações na textura dos produtos. Em estudo realizado por Hara et al. (2003), foi avaliada a aplicação de AEA e AEB em baixas concentrações em conjunto e individualmente nas propriedades oxidativas e sensoriais do Tofu, e observaram modificações no teor de proteína com a AEB em reação à AEA e água comum. Isso porque a solubilidade da proteína é geralmente melhorada quando o valor do $\mathrm{pH}$ é distante do ponto isoelétrico (PI). O pH alcalino da AEB afetou a solubilidade da proteína de soja e, portanto, alterou o teor de proteína do tofu. Isso influenciou diretamente na textura, a qual apresentou-se mais macia nas amostras com AEB, ao mesmo tempo em que houve maior retenção de água.

Além disso, estudos foram realizados em relação ao $\mathrm{NaOH}$ em emulsões cárneas. Knipe, Olson e Rust (1985) avaliaram a ação do $\mathrm{NaOH}$, em separado ou combinado com vários tipos de fosfatos na elaboração de emulsão cárnea, e constataram que a concentração de $0,075 \%$ de $\mathrm{NaOH}$ foi suficiente para aumentar o $\mathrm{pH}$ da emulsão e a proteína solubilizada, quando agindo em conjunto com o fosfato. Sobre isso, é importante ressaltar que a $\mathrm{AE}$ possui uma diversificação de aplicações, o que permite que ela seja associada a outras tecnologias alternativas, potencializando seus efeitos (FLORES et al, 2018). 
LEÃES, Y.S.; PINTON, M. B.; CORREA, L.P.; CICHOSKI, A.J.; CAMPAGNOL, P. C. B.

Nesse sentido de aplicação conjunta com outra tecnologia, Leães et al. (2020) avaliaram a ação do US em diferentes tempos em conjunto com a AEB sobre características tecnológicas e físico-químicas de emulsões cárneas com diferentes concentrações de $\mathrm{NaCl}: 2.5 \%, 2.25 \%, 2.00 \%, 1.75 \%, 1.50 \%$ e $1.25 \%$. A AEB utilizada

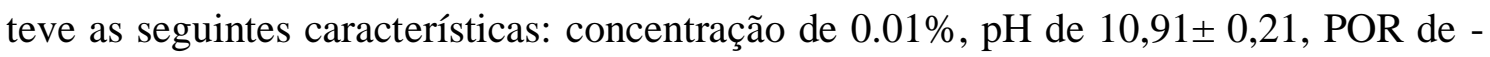
$330 \mathrm{mV} \pm 4,15$ e concentração de cloro livre (CCL) de 1,2 mg/L. Foi observado que a substituição da água por AEB na formulação possibilitou mais $10 \%$ na redução de $\mathrm{NaCl}$ em emulsão cárnea, agindo em conjunto com o US no tempo de $20 \mathrm{~min}$, que havia conferido $20 \%$ de redução. Proporcionando, assim, uma redução total de $30 \%$, considerando fatores como rendimento (\%), liberação de água e gordura (\%), textura e cor.

Outros trabalhos já evidenciaram a ação da AEB em matrizes cárneas, mas não com o propósito de redução. Vinnikova e Porkina (2016) avaliaram salmoura elaborada com AEB em relação ao rendimento em carne suína, e observaram aumento no rendimento e redução da perda de umidade em até 7,3\% após tratamento térmico quando comparado ao padrão (somente com água). Віннікова е Пронькіна (2015), ao avaliarem ação da AEB em carne moída bovina e suína, as quais posteriormente foram utilizadas para a elaboração de salsicha (utilizando a concentração de $2.5 \mathrm{de} \mathrm{NaCl}$ ), observaram redução significativa na perda de umidade do produto. Em ambos estudos, o resultado foi relacionado ao aumento do $\mathrm{pH}$ promovido pela AEB.

Acerca disso, no estudo realizado por Leães et al. (2020), três mecanismos foram identificados como possíveis causas: 1) o pH da AEB, que aumentou o pH das emulsões cárneas, distanciando-as do PI e fornecendo efeito semelhante ao do fosfato nesse sentido, auxiliando em maior retenção de água; 2) a pequena quantidade de cloro livre presente na $\mathrm{AEB}$, que pode ter auxiliado a potencializar a solubilização das proteínas; 3) e o $\mathrm{NaOH}$ presente, que poderia ter atuado na solubilização das proteínas e também em modificações nos glóbulos de gordura, visto que a redução na liberação de gordura foi significativamente $(\mathrm{p}<0.001)$ menor em relação aos demais tratamentos somente com água na formulação e aplicação de US (BAI; NYU; XIANG, 2019; XARGAIÓ et al., 2006; KONOMATSU, 2003).

Decorrente que a aplicação de AEB em alimentos ainda é pouco explorada, observa-se a necessidade de investigar qual a ação desta em produtos cárneos, uma vez que, conforme os trabalhos mencionados, o seu elevado $\mathrm{pH}$, bem como a presença de $\mathrm{NaOH}$ e cloro, podem trazer efeitos benéficos a este tipo de produto no que diz respeito a rendimento e textura, aspectos de extrema importância na viabilidade de utilização de novas tecnologias. Entretanto, é importante também investigar a ação da AEB em 
LEÃES, Y.S.; PINTON, M. B.; CORREA, L.P.; CICHOSKI, A.J.; CAMPAGNOL, P. C. B.

conjunto ou em separado ao US que diz respeito às modificações nas características microbiológicas, oxidativas e sensoriais desses produtos

\section{Considerações Finais}

As tecnologias de APH, CEP, US e AEB apresentaram efeitos expressivos nas características físico-químicas, tecnológicas, microbiológicas, oxidativas e sensoriais de produtos cárneos com teor reduzido de $\mathrm{NaCl}$ e fosfato, constituindo-se como alternativas viáveis na promoção da saudabilidade desses produtos. Elas podem ser úteis para promover diversas modificações na estrutura de matrizes cárneas, a começar pelas proteínas, contribuindo diretamente no aumento do rendimento do produto final, o qual é um aspecto de extrema importância quanto a viabilidade na aplicação.

Além disso, os estudos apresentados não evidenciaram alterações na estabilidade oxidativa, e também demonstraram que essas tecnologias tem ação benéfica sobre as características microbiológicas (eliminando microrganismos deteriorantes) e sensoriais. Entretanto, recomenda-se a continuidade nesses estudos, a fim de complementar aspectos que faltaram serem elucidados.

Em relação à $\mathrm{APH}$, necessita-se ampliar os estudos em relação à redução de fosfato; o CEP necessita ser mais explorado em relação aos efeitos que ele pode proporcionar aos produtos cárneos como presunto, produtos marinados, emulsões cárneas e etc., bem como verificar se esse efeito ocorre também em relação à redução de fosfato. Os estudos voltados para o uso do US são os que mais avançaram, pois já se tem resultados sobre sua ação em diferentes matrizes cárneas e em relação ao fosfato em emulsões, o que poderia ser aplicado em outros produtos a fim de verificar se também há eficácia. Em relação à ação da AEB, entende-se que ela consiste em uma tecnologia complementar. Entretanto, ainda é necessário dar prosseguimento ao estudo com as emulsões, avaliando a ação dela em separado ou em conjunto ao US sobre as características microbiológicas, estabilidade oxidativa e qualidade/perfil sensorial.

\section{Referências}

ALARCON-ROJO A.D.; CARRILLO-LOPEZ L.M.; REYES-VILLAGRANA R.; HUERTA-JIMÉNEZ M.; GARCIA-GALICIA I.A. 2019. Ultrasound and meat quality: A review. Ultrasonics - Sonochemistry, v. 55, p. 369-382, 2019.

AFARI, G. K.; HUNG, Y. C. 2018. A meta-analysis on the effectiveness of electrolyzed water treatments in reducing foodborne pathogens on different foods. Food Control, v. 93, p. 150-164, 2018. 
AMIRI A.; SHARIFIAN P.; SOLTANIZADEH N. 2018. Application of ultrasound treatment for improving the physicochemical, functional and rheological properties of myofibrillar proteins. International Journal of Biological Macromolecules, v. 111, p. 139-147, 2018.

ANDREU V.; DIMOPOULOS G.; ALEXANDRAKIS Z.; KATSAROS G.; OIKONOMOU D.; TOEPFL S.; HEINZ V.; TAOUKIS P. 2016. Shelf-life evaluation of virgin olive oil extracted from olives subjected to nonthermal pretreatments for yield increase. Innovative Food Science and Emerging Technologies, v. 40, p. 52-57, 2016.

ATHAYDE, D. R.; FLORES, D. R. M.; SILVA, J. S.; GENRO, A. L. G.; SILVA, M. S.; KLEIN, B.; MELLO, R.; CAMPAGNOL, P. C. B.; WAGNER, R.; MENEZES, C. R.; BARIN, J. S.; CICHOSKI, A. C. 2017. Application of electrolyzed water for improving pork meat quality. Food Research International, v. 100, p. 757-763, 2017.

ATHAYDE, D. R.; FLORES, D. R. M.; SILVA, J. S.; GENRO, A. L. G.; SILVA, M. S.; CAMPAGNOL, P. C. B.; WAGNER, R.; MENEZES, C. R.; CICHOSKI, A. C. 2018. Characteristics and use of electrolyzed water in food industries. International Food Research Journal, v. 25, n. 1, p. 11-16, 2018.

BAI, Y.; NIU, L.; XIANG, Q. 2019. Application of Electrolyzed Water in Red Meat and Poultry Processing. Chapter 5. In: DING, T.; OH, D.; LIU, D. (org.). Electrolyzed Water in Food: Fundamentals and Applications. Hangzhou: Springer, Singapore, 274p, 2019.

BARBA F.J.; KOUBAA M.; PRADO-SILVA L.; ORLIEN V.; SANT'ANA A.S. 2017. Mild processing applied to the inactivation of the main foodborne bacterial pathogens: A review. Trends in Food Science \& Technology, v. 66, p. 20-35, 2017.

BARRETO T.L.; POLLONIO M.A.R.; TELIS-ROMERO J.; BARRETO A.C.S. 2018. Improving sensory acceptance and physicochemical properties by ultrasound application to restructured cooked ham with salt $(\mathrm{NaCl})$ reduction. Meat Science, v. 145, p. 55-62, 2018.

BEKHIT A.E.D.A.; SUWANDI V.; CARNE A.; VEM R.; HOPKINS D.L. 2016. Effect of repeated pulsed electric field treatment on the quality of hot-boned beef loins and topsides. Meat Science, v. 111, p. 139-144, 2016.

BHAT Z.F.; MORTON J.D.; MANSON S.L.; BEKHIT A.E.D.A. 2018. Current and future prospects for the use of pulsed electric field in the meat industry. Critical Reviews in Food Science and Nutrition, v. 59, p. 1660-1674, 2018.

BHAT Z.F.; MORTON J.D.; MASON S.L.; BEKHIT A.E.D.A. 2019. Pulsed electric field operates enzymatically by causing early activation of calpains in beef during ageing. Meat Science, v. 153, p.144-151, 2019.

BHAT Z.F.; MORTON J.D.; MASON S.L.; BEKHIT A.E.D.A. 2020. The application of pulsed electric field as a sodium reducing strategy for meat produts. Food Chemistry, v. 306, 2020.

ВІННІКОВА, Л. Г., ПРОНЬКІНА К. В. 2015. Вплив фракцій електроактивованої води на функціональнотехнологічні властивості яловичини та свинини. ВосточноЕвропейский журнал передовых технологий, v. 75, p. 36-43, 2015. 
BUCKOW R.; CHANDRY P.S.; NG S. Y.; MCAULEY C.M.; SWANSON B.G. 2015. Opportunities and challenges in pulsed electric field processing of dairy products. International Dairy Journal, v. 34, p.199-212, 2015.

CHEMAT, F.; ZILL-E-HUMA; KHAN, M. K. 2011. Applications of ultrasound in food technology: Processing, preservation and extraction. Ultrasonics Sonochemistry, v. 18, n. 4, p. 813-835, 2011.

CHEN, F.; ZHANG M.; YANG C. 2020. Application of ultrasound technology in processing of ready-to-eat fresh food: A review. Ultrasonics Sonochemistry, v. 63, 2020.

CICHOSKI A.J.; SILVA M.S.; LEÃES Y.S.V.; BRASIL C.C.B.; MENEZES CR, BARIN J.S.; WAGNER R.; CAMPAGNOL P.C.B. 2019. Ultrasound: A promising technology to improve the technological quality of meat emulsions. Meat Science, v. 148, p. 150-155, 2019.

CUI, X.; YUCHAO, S.; ZHENGXIANG, S.; HONGWEI, X.; WEI, C. 2009. Physicochemical properties and bactericidal efficiency of neutral and acidic electrolyzed water under diferente different storage conditions. Journal of Food Engineering, v. 91, n. 4, p. 582-6, 2009.

ESPINA L.; MONFOT S.; ÁLVARES I.; GARCÍA-GONZALO D.; PAGÁN R. 2014. Comnination of pulsed electric fields, mild heat and essential oils as na alternative to the ultrapasteurization of liquid whole egg. International Journal of Food Microbiology, v. 189, p. 119-125, 2014.

FLORES, D. R. M. et al. 2018. Application of ultrasound in chicken breast during chilling by

immersion promotes a fast and uniform cooling. Food Research International, v. 109, p. 59-64, 2018.

GABRIĆ D.; BARBA F.; ROOHINAJAD S.; GHARIBZAHEDI S.M.T.; RADOJČIN M.; PUTNIK P.; KOVAČEVIĆ D.B. 2017. Pulsed electric fields as na alternative to termal processing for preservation of nutritive and physicochemical properties of beverages: A review. Journal of Food Process Engineering, v. 41, p. 1-14, 2017.

GÓMEZ B.; MUNEKATA P.E.S.; GAVAHIAN M.; BARBA F.J.; MARTÍ-QUIJAL F.J.; BOLUMAR T.; CAMPAGNOL P.C.B.; TOMASEVIC I.; LORENZO J.M. 2019. Application of pulsed electric fields in meat and fish processing industries: A review. Food Research International, v. 123, p. 95-105, 2019.

GONÇALVES C.; ABREU S.; PADRÃO P.; PINHO O.; GRAÇA P.; BREDA J.; SANTOS R.; MOREIRA P. 2016. Sodium and potassium rinary excretion and dietary intake: a cross-sectional analysis in adolescentes. Food \& Nutrition Research, v. 60, p. $1-11$.

GONZALEZ-GONZALEZ, L.; ALARCON-ROJO, A. D.; CARRILLO-LOPEZ, L. M.; GARCIA-GALICIA, I. A.; HUERTA-JIMENEZ, M.; PANIWNYK, L. 2020. Does ultrasound equally improve the quality of beef? An insight into longissimus lumborum, infraspinatus and cleidooccipitalis. Meat Science, v. 160, 2020. 
GUILLOU S.; LERASLE M.; SIMONIN H.; FEDERIGHI M. 2016. High-pressure processing of meat and meat products. In: CUMMINS, E. J.; LYNG, J. G. (Org.). Emerging Technologies in Meat Processing: Production, Processing and Technology. Edited by Cummins EJ, Lyng JG. John Wiley \& Sons, p. 37-101, 2016.

HARA, Y. H.; ATSUDA, H. M.; RAI, E. A. 2003. Effects of Weakly Electrolyzed Water on Properties of Tofu (Soybean Curd). Food science and technology research, v. 9, n. 4, p.

$332-337,2003$.

HUANG H. W.; WU S. J.; LU J. K.; SHYU Y. T.; WANG C. Y. 2017. Current status and future trends of high-pressure processing in food industry. Food Control, v. 72, p. 1 $-8,2017$.

HUANG, Y. R.; HUNG, Y.; HSU, S.; HUANG, Y.; HWANG, D. 2008. Application of electrolyzed water in the food industry. Food Control, v. 19, n. 4, p. 329-345, 2008.

INGUGLIA, E. S.; ZHANG, Z.; TIWARI, B. K.; KERRY, J. P.; BURGESS, C. M. 2017. Salt reduction strategies in processed meat products - A review. Trends in Food Science and Technology, 59, 70-78, 2008.

JADEJA, R.; HUNG, Y. C. 2014. Efficacy of near neutral and alkaline pH electrolyzed oxidizing waters to control Escherichia coli O157: H7 and Salmonella Typhimurium DT 104 from beef hides. Food Control, v. 41, n. 1, p. 17-20, 2014.

JUNIOR L. M.; ANJOS C. A. R. 2018. Effect of high-pressure processing on characteristics of flexible packaging for foods and beverages. Food Research International, v. 119, p. 920-930, 2018.

KRASULYA O.; TSIRULNICHENKO L.; POTOROKO I.; BOGUSH V.; NOVIKOVA Z.; SERGEEV A.; KUZNETSOVA T.; ANANDAN S. 2019. The study of changes in raw meat salting using acoustically activated brine. Ultrasonics Sonochemistry, v. 50, p. 224-229, 2019;

KNIPE, C. L.; OLSON, D. G.; RUST, R. E. 1985. Effects of Selected Inorganic Phosphates,

Phosphate Levels and Reduced Sodium Chloride Levels on Protein Solubility, Stability and

pH of Meat Emulsions. Journal of Food Science, v. 50, n. 4, p. 1010-1013, 1985.

KONOMATSU, A.; SUGIBAYASHI, K.; OKAJIMA, M.; ISHII, F. 2003. Preparation and stability of surfactant free emulsions using electrolyzed deoxidized and ionized water. Mater.

Technol., v. 21, p. 273-285, 2003.

LEÃES Y.S.V.; PINTON M.B.; ROSA C.T.A.; ROBALO S.S.; WAGNER R.; MENEZES C.R.; BARIN J.S.; CAMPAGNOL P.C.B.; CICHOSKI A.J. 2020. Ultrasound and basic electrolyzed water: A green approach to reduce the technological defects caused by $\mathrm{NaCl}$ reduction in meat emulsions. Ultrasonics Sonochemistry, v. 61, 2020 .

LEE, N. Y. KIM, N. H.; JANG, S.; SANG, J. H.; LEE, S. H.; HWANG, I. G.; RHEE, M. S. 2014. Decontamination efficacy of neutral electrolyzed water to eliminate indigenous 
flora on a large-scale of cabbage and carrot both in the laboratory and on a real processing line. Food Research International, v. 64, p. 234-240, 2014.

LI, J.; DING, T.; LIAO, X.; CHEN, S.; YE, X.; LIU, D. 2017. Synergetic effects of ultrasound and slightly acidic electrolyzed water against Staphylococcus aureus evaluated by flow cytometry and electron microscopy. Ultrasonics Sonochemistry, v. 38, p. 711$719,2017$.

MCDONNELL C. K.; ALLEN P.; CHARDONNEREAU F. S.; ARIMI J. M.; LYNG J. G. 2014. The use of pulsed electric fields for accelerating the salting of pork. LWT Food Science and Technology, v. 59, p. 1054 - 1060, 2014.

NOWAK K.W.; ROPELEWSKA E.; BEKHIT A.E.D.A.; MARKOWSKI M. 2017. Ultrasound applications in the meat industry. In: Advances in Meat Processing Technology. Edited by Bekhit EDA. CRC Pres, p. 3-32, 2017.

O'NEIL C.M.; CRUZ-ROMERO M.C.; DUFFY G.; KERRY J.P. 2018. Shelf life extension of vacuum-packed salt reduced frankfurters and cooked ham through the combined application of high pressure processing and organic acids. Food Packaginf and Shelf Life, v. 17, p. 120-128, 2018.

O’NEIL C.M.; CRUZ-ROMERO M.C.; DUFFY G.; KERRY J.P. 2018. The application of response surface methodology for the development of sensory accepted low-salt cooked ham using high pressure processing and a mix of organic acids. Innovative Food Sceince \& Emerging Technologies, v. 45, p. 401-411, 2018.

O'FLYNN C.C.; CRUZ-ROMERO M.C.; TROY D.; MULLEN A.M.; KERRY J.P. 2014. The application of high-pressure treatment in the reduction of salt levels in reducedphosphate breakfast sausages. Meat Science, v. 96, p. 1266-1274, 2014.

OSTERMEIER R.; GIERSEMEHL P.; SIEMER C.; TOPFL S.; JA“GER H. 2018. Influence of pulsed electric field (PEF) pre-treatment on the convective drying kinetics of onions. J Food Eng, v. 237, p. 110-117, 2018.

OVISSIPOUR, M.; AL-QADIRI, H. M.; SABLANI, S. S.; GOVIDAN, B. N.; ALALAMI, N.; RASCO, B. 2015. Efficacy of acidic and alkaline electrolyzed water for inactivating Escherichia coli O104: H4, Listeria monocytogenes, Campylobacter jejuni, Aeromonas hydrophila, and Vibrio parahaemolyticus in cell suspensions. Food Control, v. 53, p. 117- 123, 2015.

PIETRASIK Z.; GAUDETTE N.J.; JOHNSTON S.P. 2016. The use of high pressure processing to enhance the quality and shelf life of reduced sodium naturally cured restructured cooked hams. Meat Science, v. 116, p. 102 - 109, 2016.

PIETRASIK Z.; GAUDETTE N.J.; JOHNSTON S.P. 2017. The impact of high hydrostatic pressure on the functionality and consumer acceptability of reduced sodium naturally cured wieners. Meat Science, v. 129, p.127-134, 2017.

PINTON, M. B.; SANTOS, B. A.; LORENZO, J. M.; CICHOSKI, A. J.; BOEIRA, C. P.; CAMPAGNOL, P. C. 2021. Green technologies as a strategy to reduce $\mathrm{NaCl}$ and phosphate in meat products: an overview. Current Opinion in Food Science, v. 40, p. $1-5,2021$. 
PINTON, M. B.; SANTOS, B. A.; CORREA, L. P.; LEÃES, Y. S. V.; CICHOSKI, A. J.; LORENZO, J. M.; SANTOS, M.; POLLONIO, M. A. R.; CAMPAGNOL, P. C. B. 2020. Ultrasound and low-levels of $\mathrm{NaCl}$ replacers: A successful combination toproduce lowphosphate and low-sodium meat emulsions. Meat Science, v. 170, 2020.

PINTON M.B.; CORREA L.P.; FACCHI M.M.X.; HECK R.T.; LEÃES Y.S.V.; CICHOSKI A.J.; LORENZO J.M.; SANTOS M.; POLLONIO M.A.P.; CAMPAGNOL P.C.B. 2019. Ultrasound: A new approach to reduce phosphate contente of meat emulsions. Meat Science, v. 152, p. 88-95, 2019.

POTTIER L.; VILLAMONT G.; LAMBALLERIE M. 2017. Applications of high pressure for healthier foods. Current Opinion in Food Science, v. 16, p. 21 - 27, 2017.

RAHMAN, S. M. E.; JIN, Y. G.; OH, D. H. 2010. Combined Effects of Alkaline Electrolyzed

Water and Citric Acid with Mild Heat to Control Microorganisms on Cabbage. Journal of

Food Science, v. 75, n. 2, p. 111-115, 2010.

RICCI A.; PARPINELLO G.P.; VERSARI A. 2018. Recent advances and applications of pulsed electric fields (PEF) to improve polyphenol extraction and color release during red winemaking. Beverages, v. 4, p. 1-12, 2018.

SAULIS G. 2010. Electroporation of ceel membranes: The fundamental effects of pulsed electric fields in food processing. Food Engineering Reviews, v. 2, p. 52 - 73, 2010.

SHARMA P.; BREMER P.; OEY I.; EVERETT D.W. 2014. Bacterial inactivation in whole milk using pulsed electric field processing. International Dairy Journal, v. 35, p. 49-56, 2014.

SHENG, X.; SHU, D.; TANG, X.; ZANG, Y. 2018. Effects of slightly acidic electrolyzed water on the microbial quality and shelf life extension of beef during refrigeration. Food Science and Nutrition, v. 6, n. 7, p. 1975-1981, 2018.

SHIMAMURA, Y.; SHINKE, M.; HIRAISHI, M.; TSCHUIYA, Y.; MASUDA, S. 2016. The application of alkaline and acidic electrolyzed water in the sterilization of chicken breasts and beef liver. Food Science \& Nutrition, v. 41, n. 3, p. 431-440, 2016.

SIRÓ I.; VÉN C.; BALLA C.; JÓNÁS G.; ZEKE I.; FRIEDRICH L. 2009. Application of na ultrasonic assisted curing technique for improving the diffusion of sodium chloride in porcine meat. Journal of Food Engineering, v. 91, p. 353-362, 2009.

SITZMANN W.; VOROBIEV E.; LEBOVKA N. 2016. Applications of electricity and specifically pulsed electric fields in food processing: Historical backgrounds. Innovative Food Science and Emerging Technologies, v. 37, p. 302-311, 2016.

SOLADOYE O. P.; PIETRASIK Z. 2018. Utilizing high pressure processing for extended shelf life meat products. Reference Module in Food Science, p. 1-8, 2018.

SUMAN, S. P.; JOSEPH, P. 2012. Myoglobin Chemistry and Meat Color. Annual Review of Food Science and Technology, v. 4, n. 1, p. 79-99, 2012. 
TAMM A.; BOLUMAR T.; BAJOVIC B.; TOEPFL S. 2016. Salt (NaCl) reduction in cooked ham by a combined approach of high pressure treatment and the salt replacer $\mathrm{KCl}$. Innovative Food Science and Emerging Technologies, v. 36, p. 294-302, 2016.

THANGAVELU, K. P.; KERRY, J. P.; TIWARI, B. K.; MCDONNELL, C. K. 2019. Novel processing technologies and ingredient strategies for the reduction of phosphate additives in processed meat. Trends in Food Science and Technology, v. 94, p. 43-53, 2019.

TOEPFL S.; SIEMER C.; SALDAÑA-NAVARRO G.; HEINZ V. 2014. Overview of pulsed electric fields processing dor food. Emerging Technologies for Food Processing, v. 2, p. 93-114, 2014.

VINNIKOVA, L. G.; PRONKINA, K. V. 2016. The changes of characteristics of the pork whole muscle meat products while using the electrolyzed water. Харчова наука i технологія, v. 10, n. 2, p. 19-23, 2016.

XARGAIÓ, M.; LAGARES, J.; FERNÁNDES, E.; BORREL, D.; JUNCÁ, G. 2006. Una solución definitive para mejorar la textura de la carne. Departamento Tecnológico de Metalquimia, 2006.

WANG, H.; QI, J.; DUAN, D.; DONG, Y.; XU, X.; ZHOU, G. 2019. Primary concerns regarding the application of electrolyzed water in the meat industry. Food Control, v. 95, p. $50-56,2019$.

WANG Y.; ZHOU Y.; LI P.; WANG X.; CAI K.; CHEN C. 2018a. Combined effect of $\mathrm{CaCl} 2$ and high pressure processing on the solubility of chicken breast myofibrillar proteins under sodium-reduced conditions. Food Chemistry, v. 269, p. 236-243, 2018 a.

WANG, H.; QI, J.; DUAN, D.; DONG, Y.; XU, X.; ZHOU, G. 2018b. Combination of a novel designed spray cabinet and electrolyzed water to reduce microorganisms on chicken carcasses. Food Control, v. 86, p. 200-206, 2018 b.

YANG N.; HUANG K.; LYU C.; WANG J. 2016. Pulsed electric field technology in the manufacturing processes of wine, beer, and rice wine: A review. Food Control, v. 61, p. $28-38,2016$.

YOGESH K. 2015. Pulsed electric field processing of egg products: A review. Journal Food Science Technology, v. 53, p. 934-945, 2015.

ZHENG H.; HAN M.; YANG H.; TANG C.; XU X.; ZHOU G. 2017. Application of high pressure to chicken meat batters during heating modifies physicochemical properties, enabling salt reduction for high-quality products. LWT - Food Science and Technology, v. 84, p. 693-700, 2017. 